\title{
Utility of ultrasound joint counts in the prediction of rheumatoid arthritis in patients with very early synovitis
}

\author{
Andrew Filer, 1,2 Paola de Pablo, 1,2 Gina Allen, ${ }^{3}$ Peter Nightingale, ${ }^{4}$ Alison Jordan, ${ }^{5}$ \\ Paresh Jobanputra, ${ }^{5}$ Simon Bowman, ${ }^{5}$ Christopher D Buckley, ${ }^{1,2}$ Karim Raza ${ }^{1,2}$
}

\begin{abstract}
- Additional data
(supplementary tables and figures) are published online only. To view these files please visit the journal online (http:// ard.bmj.com).
\end{abstract}

${ }^{1} \mathrm{MRC}$ Centre for Immune Regulation, School of Immunity and Infection, The University of Birmingham, Birmingham, UK ${ }^{2}$ Department of Rheumatology, Sandwell and West Birmingham Hospitals NHS Trust,

Birmingham, UK

${ }^{3}$ Green Templeton College,

University of Oxford, Oxford, UK

${ }^{4}$ Wolfson Computer Laboratory, University Hospital Birmingham NHS Foundation Trust,

Birmingham, UK

${ }^{5}$ Department of Rheumatology, University Hospitals Birmingham NHS Foundation Trust, Birmingham, UK

\section{Correspondence to}

Dr A Filer, Rheumatology Research Group, School of Immunity and Infection, College of Medical and Dental Sciences, Institute for Biomedical Research, The University of Birmingham, Birmingham B15 2TT, UK; a.filer@bham.ac.uk

Accepted 17 October 2010 Published Online First 29 November 2010

\section{(6) UNLOCKED}

This paper is freely available online under the BMJ Journals unlocked scheme, see http:// ard.bmj.com/info/unlocked.dtl

\begin{abstract}
Objectives Early therapy improves outcomes in rheumatoid arthritis (RA). It is therefore important to improve predictive algorithms for RA in early disease. This study evaluated musculoskeletal ultrasound, a sensitive tool for the detection of synovitis and erosions, as a predictor of outcome in very early synovitis.
\end{abstract}

Methods 58 patients with clinically apparent synovitis of at least one joint and symptom duration of $\leq 3$ months underwent clinical, laboratory, radiographic and 38 joint ultrasound assessments and were followed prospectively for 18 months, determining outcome by 1987 American College of Rheumatology (ACR) and 2010 ACR/European League Against Rheumatism criteria. Sensitivity and specificity for 1987 RA criteria were determined for ultrasound variables and logistic regression models were then fitted to evaluate predictive ability over and above the Leiden rule.

Results 16 patients resolved, 13 developed non-RA persistent disease and 29 developed RA by 1987 criteria. Ultrasound demonstrated subclinical wrist, elbow, knee, ankle and metatarsophalangeal joint involvement in patients developing RA. Large joint and proximal interphalangeal joint ultrasound variables had poor predictive ability, whereas ultrasound erosions lacked specificity. Regression analysis demonstrated that greyscale wrist and metacarpophalangeal joint involvement, and power Doppler involvement of metatarsophalangeal joints provided independently predictive data. Global ultrasound counts were inferior to minimal power Doppler counts, which significantly improved area under the curve values from 0.905 to 0.962 combined with the Leiden rule.

Conclusion In a longitudinal study, extended ultrasound joint evaluation significantly increased detection of joint involvement in all regions and outcome groups. Greyscale and power Doppler scanning of metacarpophalangeal joints, wrists and metatarsophalangeal joints provides the optimum minimal ultrasound data to improve on clinical predictive models for RA.

Early therapy significantly improves outcomes in rheumatoid arthritis (RA). ${ }^{1}{ }^{2}$ Indeed, data suggest that the first 3 months after symptom onset may represent a pathologically distinct phase that translates into a therapeutic window of opportunity. ${ }^{3-5}$ The ability to predict the development of RA accurately in patients with very early synovitis is thus important. ${ }^{6}$

Classification systems for $\mathrm{RA}^{7} 8$ and predictive models such as the widely validated Leiden rule, ${ }^{910}$ rely heavily on clinical assessment of the extent and pattern of joint involvement. How best to define early RA remains a subject of considerable debate $^{11}$ heightened by recent publication of the 2010 American College of Rheumatology (ACR)/ European League Against Rheumatism (EULAR) criteria. Musculoskeletal ultrasound has been demonstrated to be more sensitive than clinical assessment in the detection of joint swelling,,$^{12}$ and more sensitive than conventional radiography in the detection of erosions. ${ }^{14}$ It is therefore important to evaluate the contribution of ultrasound variables as potential predictors of outcome in patients with very early disease.

Investigators have recently explored the use of restricted ultrasound joint counts to predict persistent inflammatory arthritis in symptomatic patients with hand synovitis or arthralgia presenting in the first 3 months of disease. ${ }^{15}$ However, the use of ultrasound to predict RA in this early phase has not been investigated, and although extended joint counts are being investigated as a tool to assess response to therapy, ${ }^{16}$ they have yet to be applied to an unselected population of patients with very early synovitis. The aim of this study was therefore to evaluate the additional predictive ability of extended ultrasound joint counts for RA. We first compared clinical and ultrasound baseline assessments in very early arthritis. Second, we compared ultrasound versus a conventional radiography baseline evaluation of bone erosion. Finally, we compared ultrasound and clinical variables for their ability to predict RA as a diagnostic outcome.

\section{PATIENTS AND METHODS \\ Patients}

Fifty-eight patients with clinically apparent synovitis of at least one joint and inflammatory joint symptoms (inflammatory joint pain, and/or swelling and/or morning stiffness) of 3 months or less duration underwent baseline assessment and 18-month follow-up to determine diagnosis as previously described. ${ }^{3} 17$ Ethical permission was obtained and all patients gave written informed consent. Patients were classified as having RA, reactive arthritis, psoriatic arthritis or miscellaneous conditions according to established criteria. 818 19 In order to compare the distribution of joint involvement with established RA, 22 patients with newly presenting, treatment-naive RA of over 3 months' duration fulfilling 1987 ACR criteria were also recruited. 


\section{Clinical, laboratory and radiographic assessment}

Patients underwent baseline 66 swollen and 68 tender clinical counts. Age, sex, symptom duration, early morning stiffness duration, medication, erythrocyte sedimentation rate, C-reactive protein, rheumatoid factor (RF) and anticyclic citrullinated peptide antibody (ACPA) status were recorded. In all but six patients, none of whom fulfilled the 1987 or 2010 criteria for RA or subsequently developed erosions, baseline conventional radiography of hands and feet was recorded, and the presence of erosions assessed in a blinded fashion by a single trained observer (AF).

\section{Ultrasound assessment}

Within 24 h of clinical assessment, patients underwent blinded ultrasound assessment in a temperature controlled radiology suite. Patients were asked not to discuss their symptoms. A systematic multiplanar greyscale and power Doppler ultrasound examination of 92 sites in 38 joints (table 1) was performed based upon standard EULAR reference scans ${ }^{20}$ using a Siemens Acuson Antares scanner (Siemens, Bracknell, UK) and multifrequency $(5-13 \mathrm{MHz})$ linear array transducers. For power Doppler examinations, the pulse repetition frequency was adjusted to provide maximal sensitivity at the lowest possible value for each joint, resulting in a pulse repetition frequency of between 610 and 780. Examinations took between 50 and 60 min depending on disease extent and patient mobility.

Ultrasound findings of synovitis, power Doppler positivity and erosion were defined according to consensus definitions. ${ }^{12} 20-22$ Greyscale synovitis in metacarpophalangeal, proximal interphalangeal and metatarsophalangeal joints was graded from 0 to 3 based upon the system of Szkudlarek and colleagues, ${ }^{12}$ 23 reclassifying the equivocal 'minimal' thickening grade as normal: grade 0 , normal; grade 1 , synovial thickening bulging over the line linking the tops of the periarticular bones; grade 2 , grade 1 plus extension to one bone diaphysis; grade 3 , grade 1 plus extension to both bone diaphyses. Synovitis in other joints was graded $0-3$ as: 0 , normal; 1 , mild; 2 , moderate; and 3 , severe, in which grade 1 demonstrates synovial thickening in excess of the mean plus $2 \mathrm{SD}$ of normal range when available. ${ }^{22}$

Table 1 Synovial intra-articular recesses and periarticular sites evaluated by ultrasound

\begin{tabular}{ll}
\hline Joint & Recess or site \\
\hline MCP (1-5), PIP (1-5), MTP (2-5) & Dorsal recess \\
& Lateral recess (PIP, MCP 1,2,5 MTP 5) \\
& Volar recess (PIP) \\
Wrist & Intercarpal recesses \\
& Radiocarpal recesses \\
& Ulnarcarpal recesses \\
& Volar carpal recesses \\
Elbow & Anterior recess \\
& Humeroradial joint \\
& Humeroulnar joint \\
& Posterior recess \\
Shoulder & Subdeltoid bursa \\
& Posterior glenohumeral recess \\
Knee & Suprapatellar recess \\
& Medial parapatellar recess \\
& Lateral parapatellar recess \\
& Medial femorotibial joint line \\
& Lateral femorotibial joint line \\
Ankle & Anterior tibiotalar recess \\
& Medial tibiotalar recess \\
& Lateral tibiotalar recess \\
\hline
\end{tabular}

MCP, metacarpophalangeal joint; MTP, metatarsophalangeal joint; PIP, proximal interphalangeal joint.
Effusion in the absence of synovial thickening was not classified as synovitis. Synovial hyperaemia was measured by power Doppler in each recess and the maximal score graded according to Szkudlarek et a ${ }^{23}$ : 0, absence; 1, isolated signals; 2, confluent signals in less than half of the synovial area; and 3 , confluent signals in more than half of the synovial area. The presence of joint erosion was measured as a binary variable. Global ultrasound indices for greyscale synovitis and power Doppler were calculated by adding scores from all joints. Global ultrasound counts were calculated by adding scores after converting individual joint grades to binary variables.

\section{Statistical analysis}

Analysis of data including logistic regression was performed using Stata 10. Comparison of clinical and ultrasound counts within and between groups was analysed using McNemar's test and Fisher's exact test, respectively. Other baseline clinical and ultrasound variables were compared between groups using Mann-Whitney U or Kruskal-Wallis tests. Intraobserver reliability was evaluated by blindly rescoring representative images of 20 patients for synovitis and power Doppler at least 3 months after initial scans, and analysed using $\kappa$ statistics (see supplementary table S1, available online only).

\section{RESULTS}

\section{Patient characteristics}

Patients developing RA by 1987 criteria (VERA) were significantly older than those in other groups as expected (table 2). Male patients were overrepresented in this group (55\%) compared with the general RA population. No patients in the study had received disease-modifying antirheumatic drugs at baseline. Two patients (both of whom developed RA) had received a short course of prednisolone for 5 and 14 days before recruitment. Both fulfilled ACR criteria at the time of recruitment. Two patients in the group developing non-RA persistent disease (VENRA) were RF positive; one had reactive arthritis, the other had psoriatic arthritis. Of five VENRA patients remaining unclassified, one was treated with methotrexate and another with hydroxychloroquine within 1 month of presentation. The comparison group of 22 patients with established RA had a median symptom duration of 28 (IOR 17-65) weeks and median age 55 (IOR 45-63) years: 73\% were women, and 64\% were ACPA and/or RF positive.

\section{Global clinical and ultrasound assessment of patients}

A total of 4640 sites in 2204 joints was included in the analysis. Proportionately more joints were found to be involved by ultrasound greyscale assessment than clinical examination in VERA (see table 2). Ultrasound assessment led to the reclassification of many patients between monarthritis, oligoarthritis and polyarthritis groups. In particular nine (69\%) VENRA patients were reclassified as polyarthritis, and eight $(50 \%)$ resolving patients with a clinical monarthritis were reclassified as oligo or polyarthritis. The distribution of subclinical joint involvement found by ultrasound greyscale assessment in six patients with persistent disease and a clinical monarthritis at presentation (who without erosions could not be classified as having RA by the 2010 criteria) is shown in supplementary figure S1 (available online only).

Effect of ultrasound assessment on joint involvement by region Clinical involvement was defined by at least one joint in a given region being clinically swollen and ultrasound involvement 
Table 2 Baseline patient demographics and global clinical and ultrasound data

\begin{tabular}{|c|c|c|c|}
\hline Final diagnostic group by 1987 criteria & VERA & VENRA & Resolving \\
\hline $\mathrm{n}$ & 29 & 13 & 16 \\
\hline \multicolumn{4}{|l|}{ Final diagnosis, $\mathrm{n}(\%)$} \\
\hline RA & $29(100)$ & 0 & 0 \\
\hline PsA & 0 & $5(38)$ & $1(6.25)$ \\
\hline SLE & 0 & $2(15)$ & 0 \\
\hline Parvovirus related & 0 & 0 & $1(6.25)$ \\
\hline Pseudogout & 0 & 0 & $1(6.25)$ \\
\hline Reactive & 0 & $1(8)$ & 0 \\
\hline Septic & 0 & 0 & $1(6.25)$ \\
\hline Unclassified & 0 & $5(38)$ & $12(75)$ \\
\hline $\operatorname{Age}^{\dagger * *}$ & $63(19-82)$ & $45(18-83)$ & $40(23-75)$ \\
\hline Female, $n(\%)$ & $13(45)$ & $9(69)$ & $10(63)$ \\
\hline Symptoms duration, weeks ${ }^{\dagger}$ & $7(2-12)$ & $6(3-12)$ & $4(1-9)$ \\
\hline Morning stiffness, $\min ^{\dagger}$ & $120(30-360)$ & $60(0-240)$ & $52.5(0-240)$ \\
\hline NSAID use, $\mathrm{n}(\%)$ & $20(69)$ & $8(62)$ & $13(81)$ \\
\hline RF positive, $n(\%)$ & $15(52)$ & $2(15)$ & 0 \\
\hline ACPA positive, $n(\%)$ & $14(48)$ & 0 & 0 \\
\hline ESR $(\mathrm{mm} / \mathrm{h})^{\dagger}$ & $25(0-104)$ & $24(4-87)$ & $21.5(0-102)$ \\
\hline $\operatorname{CRP}(\mathrm{mg} /)^{\dagger}$ & $15(0-102)$ & $16(0-83)$ & $16(0-244)$ \\
\hline Swollen joint count of $66^{\dagger * * *}$ & $8(1-28)$ & $2(1-13)$ & $1.5(1-7)$ \\
\hline Tender joint count of $68^{\dagger *}$ & $9(0-41)$ & $3(0-19)$ & $2.5(1-10)$ \\
\hline DAS28 score ${ }^{\dagger}$ & $4.49(2.20-6.28)$ & $3.90(1.66-6.27)$ & $3.57(1.38-4.94)$ \\
\hline \multicolumn{4}{|l|}{ Patients meeting ACR criteria at baseline } \\
\hline 1987 ACR criteria, $n(\%)$ & $12(41)$ & 0 & 0 \\
\hline USGS 1987 ACR criteria, $n(\%)^{\ddagger}$ & $16(55)$ & $3(23)$ & $1(6)$ \\
\hline 2010 ACR/EULAR criteria, $n(\%)$ & $24(83)$ & $2(15)$ & 0 \\
\hline USGS 2010 ACR/EULAR criteria, $n(\%)^{\ddagger}$ & $27(93)$ & $5(38)$ & $2(13)$ \\
\hline \multicolumn{4}{|l|}{ Clinical pattern, n (\%) } \\
\hline Monarthritis & $1(3)$ & $6(46)$ & $8(50)$ \\
\hline Oligoarthritis (2-5 joints) & $10(34)$ & $6(46)$ & $7(44)$ \\
\hline Polyarthritis ( $>5$ joints) & $18(62)$ & $1(8)$ & $1(6)$ \\
\hline \multicolumn{4}{|l|}{ USGS pattern, $\mathrm{n}(\%)^{\ddagger}$} \\
\hline Monarthritis & 0 & $1(8)$ & 0 \\
\hline Oligoarthritis & $1(3)$ & $2(15)$ & $11(69)$ \\
\hline Polyarthritis & $28(97)$ & $10(77)$ & $5(31)$ \\
\hline \multicolumn{4}{|l|}{ US variables } \\
\hline GS index ${ }^{\dagger * * *}$ & $35(5-78)$ & $14(2-38)$ & $6(2-19)$ \\
\hline GS count of $38^{\dagger * * *}$ & $15(5-33)$ & $9(1-20)$ & $3(2-12)$ \\
\hline PD index ${ }^{\dagger * * *}$ & $25(5-60)$ & $9(2-26)$ & $5.5(0-16)$ \\
\hline PD count of $38^{\dagger * * *}$ & $12(3-29)$ & $5(1-13)$ & $3(0-10)$ \\
\hline \multicolumn{4}{|l|}{ Presence of erosions } \\
\hline Radiographic hand/foot erosion, $\mathrm{n}(\%)$ & $1(3.5)$ & 0 & 0 \\
\hline US hand/foot erosion, $\mathrm{n}(\%)$ & $11(38)$ & $2(15)$ & 0 \\
\hline Any US erosion, n (\%) & $11(38)$ & $2(15)$ & $1(6.25)$ \\
\hline
\end{tabular}

\footnotetext{
${ }^{\dagger}$ Median (range).

${ }^{\ddagger}$ Clinical examination variables have been extended by adding ultrasonographic criteria of joint involvement (USGS grade $\geq 1$ ) and erosion.

ACPA, anticyclic citrullinated peptide antibody; ACR, American College of Rheumatoloty; CRP, C-reactive protein; DAS28, disease activity score in 28 joints; ESR, erythrocyte sedimentation rate; EULAR, European League Against Rheumatism; GS, greyscale ultrasound; NSAID, non-steroidal anti-inflammatory drug; PD, power Doppler; PsA, psoriatic arthropathy; RA, rheumatoid arthritis; $\mathrm{RF}$, rheumatoid factor; SLE, systemic lupus erythematosus; US, ultrasound.

${ }^{*} \mathrm{p}<0.05,{ }^{* *} \mathrm{p}<0.01,{ }^{* * *} \mathrm{p}<0.001$ Kruskal-Wallis test.
}

by the presence of greyscale synovial hypertrophy of at least grade 1 . The impact of increased sensitivity of ultrasound was most marked in the large joints, wrists and metatarsophalangeal joints (figure 1, supplementary table S1, available online only). Among VERA patients, clinically silent involvement of the wrists, elbows, knees, ankles and metatarsophalangeals joints was identified significantly more often by ultrasound. In VENRA patients, metacarpophalangeal joint $(p<0.05)$, wrist $(p<0.05)$, elbow $(p<0.05)$ and metatarsophalangeal joint $(p<0.01)$ involvement was detected more often by ultrasound (supplementary table S2, available online only). Compared with groups with persistent outcomes, ultrasound detected less additional involvement in the resolving group at the wrist $(p<0.05)$ and metatarsophalangeals joints $(\mathrm{p}<0.01)$. To investigate the low levels of clinically apparent metatarsophalangeal joint and ankle synovitis in VERA patients, we assessed a comparison group of patients with newly presenting RA of more than 3 months' duration. Clinical involvement of the proximal interphalangeal, ankle and metatarsophalangeals joints was more overt in these patients (figure 1C), with significantly greater involvement of the metatarsophalangeal joints $(p<0.05)$.

\section{Detection of erosive disease in early arthritis using ultrasound}

Ultrasonographic erosions of the hands or feet were detected in 26 joints of 13 very early arthritis patients (table 2). Using conventional radiography, only one erosion was visible in the wrist 

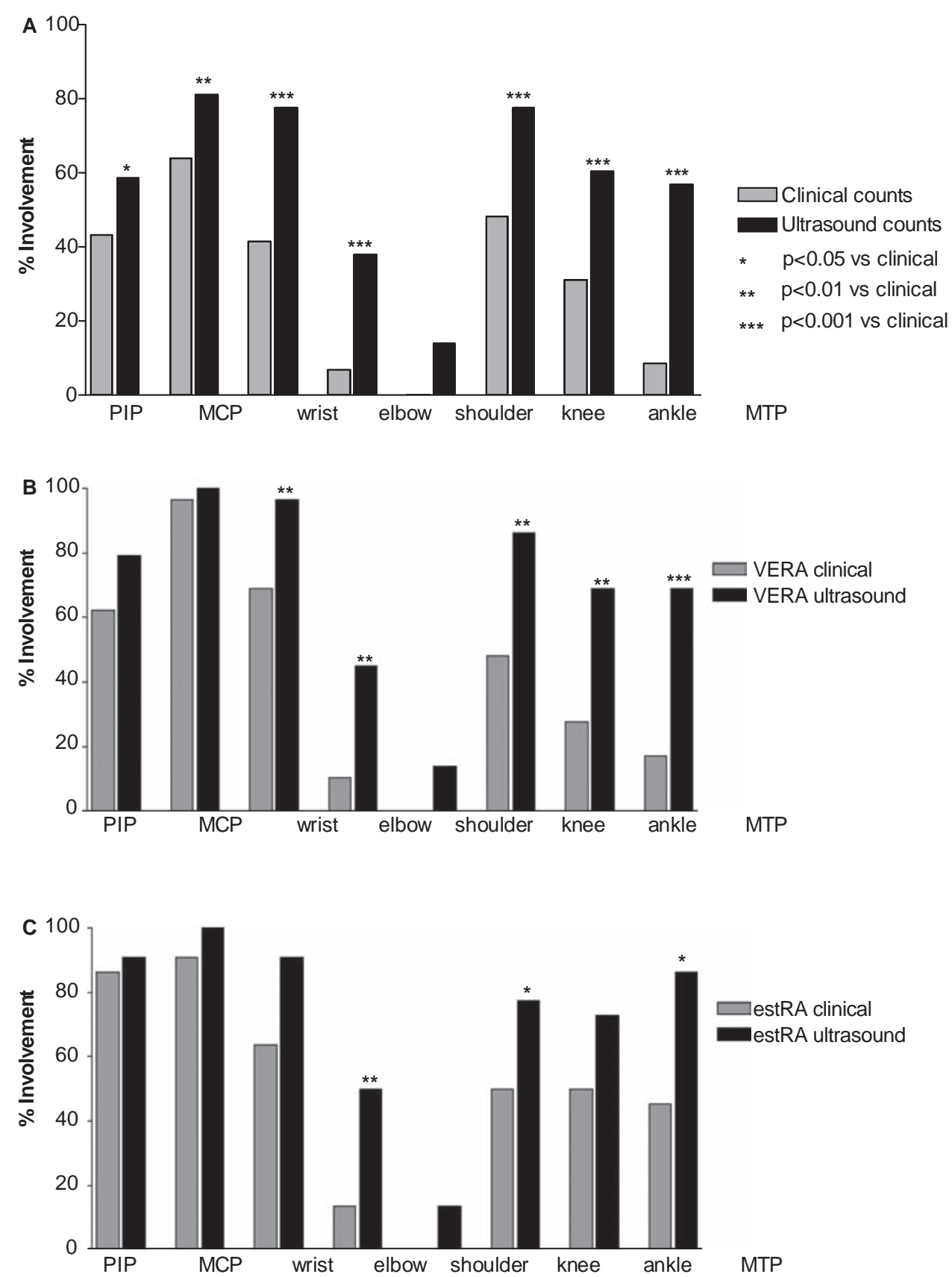

Figure 1 Clinical and ultrasound involvement by joint region. Joint region involvement, defined as the presence of at least one clinically swollen joint or one joint with ultrasound greyscale synovitis in a given region, in (A) the total cohort of very early arthritis patients, $(B)$ patients who developed a diagnosis of rheumatoid arthritis (RA) and (C) an additional cohort of patients with newly presenting, untreated RA of greater than 3 months' symptom duration. (A) Ultrasound demonstrates increased sensitivity compared with clinical examination in all joints overall. (B) Significantly more clinically silent disease in patients developing RA is measured by ultrasound at the wrist, elbow, knee, ankle and metatarsophalangeal (MTP) joints. (C) Proximal interphalangeal joint (PIP), ankle and metatarsophalangeal joint disease is clinically more overt in patients with longer RA disease duration. MCP, metacarpophalangeal joint.

of a VERA patient. All VERA patients with ultrasound erosions at joints besides metacarpophalangeals joints or wrists also had erosions at these hand joints, giving a specificity of ultrasound erosions for RA of $93 \%$. Of 11 VERA patients with ultrasound erosions, eight were RF and ACPA positive, one was RF positive only and one was seronegative. One RF-negative patient with psoriatic arthritis and one with unclassified disease presented with ultrasound wrist erosions. A single resolving patient with a diagnosis of septic arthritis presented with an ultrasound ankle erosion.

\section{Impact of ultrasound measured variables on 1987 and 2010 RA criteria fulfilment}

At baseline, the 1987 ACR criteria identified 12 out of 29 RA patients, and no patients in VENRA and resolving groups. Adding ultrasound data identified 16 RA patients but misclassified a further four patients (table 2). The 2010 criteria identified 26 patients (nine with ultrasound erosions) at baseline including two VENRA patients (table 2, supplementary table S3, available online only). The difference between these values is almost entirely accounted for by the 6-week rule, without which 1987 
criteria identified 23 VERA patients at baseline. Extending the 2010 criteria by adding ultrasound data identified a further eight patients, including three with erosions (supplementary table S2, available online only). All eight were classified as RA regardless of erosions by increasing the 2010 'joints' score from either two or three to the maximum five. Ultimately, at 18 months the 2010 criteria failed to classify as RA four out of 29 VERA patients, including one patient with ultrasound defined erosions.

\section{Sensitivity and specificity of clinical and ultrasound variables for RA by 1987 criteria}

Clinical and ultrasound variables were assessed by calculating sensitivity, specificity and area under the receiver operating characteristic curve (AUC) for 1987 RA as an outcome (table 3), using threshold grades of 1 or more or 2 or more for ultrasound variables. Shoulder, elbow, knee and ankle ultrasound involvement was discarded from the analysis because of lack of specificity for RA, despite increased sensitivity (supplementary table S2, available online only). Ultrasound variables generally improved sensitivity with some loss of specificity. Modifying this by imposing a higher threshold grade or requiring symmetry resulted in improved AUC values for ultrasound variables in metacarpophalangeal, proximal interphalangeal and metatarsophalangeal joint regions compared with clinical equivalents (table 3). In this cohort, adding ultrasound to clinical variables in the 1987 ACR criteria increased sensitivity at a cost of specificity resulting in a drop in AUC. However, power Doppler variables performed better than greyscale variables. Furthermore, greyscale and power Doppler assessments of metatarsophalangeals joints exhibited improved sensitivity compared with clinical variables, whereas power Doppler variables retained high specificity for RA. By combining variables with $100 \%$ specificity for RA (ACPA positivity, metatarsophalangeal joint power Doppler grade of 2 or more involvement and a metacarpophalangeal joint greyscale count of 8 or greater), $76 \%$ of VERA patients were identified, including eight of the 15 ACPA-negative individuals.

\section{Logistic regression analyses}

Significant variables on univariate analysis were entered as explanatory variables in logistic regression models with an outcome of RA by 1987 criteria as the dependent variable, and the Leiden score ${ }^{9}$ as the independent variable. An AUC was constructed to assess contribution to the prediction of RA above the Leiden rule for different models (table 4). The AUC for the Leiden rule as a continuous variable was 0.905 in this sample, similar to the value derived by van der Helm-van Mil et al. ${ }^{9}$ Global greyscale and power Doppler counts increased the AUC for predicting RA (table 4), indicating that ultrasound counts provide independently predictive data over and above the Leiden score.

An important aim of this study was to identify joint regions with the greatest potential for use in predictive models. We systematically examined individual ultrasound variables in combination with the Leiden rule by logistic regression (table 4). This analysis precludes examination of variables with $100 \%$ sensitivity or specificity, which were therefore omitted. No proximal interphalangeal joint ultrasound variables functioned as independent predictors. However, highly sensitive variables such as greyscale and power Doppler involvement of metacarpophalangeal joints and wrists contributed additional predictive information. Moreover, highly specific ultrasound variables such as high grade greyscale wrist symmetry, high ultrasound counts of metacarpophalangeal joints and power Doppler involvement and symmetry of metatarsophalangeal joints are most likely to suggest a diagnosis of RA if not clinically apparent, and may be combined to enhance prediction (table 3 ). Deriving a minimal 12 -joint power Doppler ultrasound score in a similar manner to Naredo et a ${ }^{24}$ increased the AUC in this analysis. By removing the (less specific) knee joint from this score, a significantly increased AUC of 0.962 was obtained ( $<<0.05$, figure 2$).{ }^{25}$

\section{DISCUSSION}

We have demonstrated a diagnostic benefit of the increased sensitivity of ultrasound in an early synovitis population. Ultrasound assessment results in a considerable shift in disease category from monarthritis to oligoarthritis and/or polyarthritis, that is greater than that reported in patients with longer disease durations. ${ }^{13}$ This suggests that the increased sensitivity of ultrasound may have a greater impact in the very early window. Comparing very early with later onset RA supported this, as joints such as the metatarsophalangeals joints were more evident clinically later in the disease course. The 2010 criteria proved, as expected, to be more sensitive at baseline than the 1987 criteria. However, they failed to classify all patients ultimately classified as RA by the 1987 criteria. Adding ultrasound variables to the new 2010 criteria classified more patients as RA, including several later classified as RA by the 1987 criteria, one with ultrasound erosions. This suggests that the detection of subclinical disease by imaging will similarly prove useful in optimising sensitivity and specificity of the 2010 criteria.

Global ultrasound counts improve sensitivity with some loss of specificity. However, ultrasound of large joints and proximal interphalangeals joints is not helpful in predicting RA by the 1987 criteria. Global ultrasound joint counts therefore increased the discriminating ability of the Leiden rule, but require significant scanning time, and performed worse than minimal counts by including non-discriminating joints. Harrison and Symmons ${ }^{26}$ showed in the NOAR cohort that persistent synovitis was predicted by the presence of RF, a tender joint count of greater than six and ankle synovitis. However, ultrasound detected significantly more knee and ankle disease in all disease groups in our cohort, with no predictive benefit.

Two further findings from our data are important: first, that scanning the metacarpophalangeals joints, wrists and metatarsophalangeal joints is likely to be of useful predictive value. Subclinical ultrasound metatarsophalangeal joint involvement in very early arthritis demonstrated very good specificity for RA by the 1987 criteria. Long established data show that erosive metatarsophalangeal joint disease occurs despite the absence of symptoms or signs. ${ }^{27}$ Such subclinical disease may manifest as a positive metatarsophalangeal joint squeeze test, a suggested screening tool for possible early RA. ${ }^{28}$ Second, power Doppler measurements have a uniquely high specificity for RA compared with other groups, particularly at the metatarsophalangeals joints, with combined metacarpophalangeal joint, wrist and metatarsophalangeal joint assessments providing excellent AUC values. These data are compatible with those of Freeston et al ${ }^{15}$ who examined associations of inflammatory arthritis in a mixed population of patients with arthralgia and arthritis, finding that high grade power Doppler had good sensitivity and specificity for persistence. Therefore, in addition to correlations with erosive progression, power Doppler may also have useful predictive power for RA. ${ }^{29} 30$ Of particular interest is the increased AUC value of power Doppler indices and counts compared with greyscale equivalents, suggesting that power Doppler has superior specificity for RA. Reducing the complexity of power 
Table 3 Sensitivity and specificity of clinical and ultrasound variables for the prediction of RA

\begin{tabular}{|c|c|c|c|c|c|c|c|}
\hline & Sensitivity & Specificity & Positive LR & Negative LR & PPV & NPV & AUC \\
\hline \multicolumn{8}{|l|}{$\mathrm{MCP}$ joints } \\
\hline MCP clinical involvement & 97 & 69 & 3 & 0.05 & 76 & 95 & 0.828 \\
\hline MCP clinical symmetry & 65.5 & 97 & 19 & 0.36 & 95 & 74 & 0.810 \\
\hline MCP GS $\geq 1$ involvement & 100 & 38 & 2 & 0 & 62 & 100 & 0.690 \\
\hline MCP GS $\geq 1$ symmetry & 93 & 76 & 4 & 0.09 & 79 & 92 & 0.845 \\
\hline MCP GS $\geq 2$ involvement & 90 & 48 & 2 & 0.21 & 63 & 82 & 0.690 \\
\hline MCP GS $\geq 2$ symmetry & 86 & 79 & 4 & 0.17 & 81 & 85 & 0.828 \\
\hline MCP GS count $\geq 8$ & 45 & 100 & & 0.55 & 100 & 64 & 0.724 \\
\hline MCP PD $\geq 1$ involvement & 97 & 55 & 2 & 0.06 & 68 & 94 & 0.759 \\
\hline MCP PD $\geq 1$ symmetry & 83 & 79 & 4 & 0.22 & 80 & 82 & 0.810 \\
\hline MCP PD $\geq 2$ involvement & 90 & 66 & 3 & 0.16 & 72 & 86 & 0.776 \\
\hline MCP PD $\geq 2$ symmetry & 83 & 83 & 5 & 0.21 & 83 & 83 & 0.828 \\
\hline \multicolumn{8}{|l|}{ PIP joints } \\
\hline PIP clinical involvement & 62 & 76 & 3 & 0.50 & 72 & 67 & 0.690 \\
\hline PIP clinical symmetry & 41 & 93 & 6 & 0.63 & 86 & 61 & 0.672 \\
\hline PIP GS $\geq 1$ involvement & 79 & 62 & 2 & 0.33 & 68 & 75 & 0.707 \\
\hline PIP GS $\geq 1$ symmetry & 48 & 79 & 2 & 0.65 & 70 & 61 & 0.638 \\
\hline PIP GS $\geq 2$ involvement & 79 & 66 & 2 & 0.32 & 70 & 76 & 0.724 \\
\hline PIP GS $\geq 2$ symmetry & 48 & 90 & 5 & 0.58 & 82 & 63 & 0.690 \\
\hline PIP PD $\geq 1$ involvement & 76 & 69 & 2 & 0.35 & 71 & 74 & 0.724 \\
\hline PIP PD $\geq 1$ symmetry & 45 & 90 & 4 & 0.62 & 81 & 62 & 0.672 \\
\hline PIP PD $\geq 2$ involvement & 66 & 76 & 3 & 0.46 & 73 & 69 & 0.707 \\
\hline PIP PD $\geq 2$ symmetry & 38 & 97 & 11 & 0.64 & 92 & 61 & 0.672 \\
\hline \multicolumn{8}{|l|}{ Wrist joints } \\
\hline Wrist clinical involvement & 69 & 86 & 5 & 0.36 & 83 & 74 & 0.776 \\
\hline Wrist clinical symmetry & 48 & 93 & 7 & 0.56 & 88 & 64 & 0.707 \\
\hline Wrist GS $\geq 1$ involvement & 97 & 41 & 2 & 0.08 & 62 & 92 & 0.690 \\
\hline Wrist GS $\geq 1$ symmetry & 86 & 62 & 2 & 0.22 & 69 & 82 & 0.741 \\
\hline Wrist GS $\geq 2$ involvement & 79 & 69 & 3 & 0.30 & 72 & 77 & 0.741 \\
\hline Wrist GS $\geq 2$ symmetry & 52 & 93 & 7 & 0.52 & 88 & 66 & 0.724 \\
\hline Wrist $\mathrm{PD} \geq 1$ involvement & 93 & 48 & 2 & 0.14 & 64 & 88 & 0.707 \\
\hline Wrist $P D \geq 1$ symmetry & 83 & 66 & 2 & 0.26 & 71 & 79 & 0.741 \\
\hline Wrist $P D \geq 2$ involvement & 90 & 48 & 2 & 0.21 & 63 & 82 & 0.690 \\
\hline Wrist $P D \geq 2$ symmetry & 69 & 72 & 2 & 0.43 & 71 & 70 & 0.707 \\
\hline \multicolumn{8}{|l|}{ MTP joints } \\
\hline MTP clinical involvement & 17 & 100 & & 0.83 & 100 & 55 & 0.586 \\
\hline MTP clinical symmetry & 10 & 100 & & 0.90 & 100 & 53 & 0.552 \\
\hline MTP GS $\geq 1$ involvement & 69 & 55 & 2 & 0.56 & 61 & 64 & 0.621 \\
\hline MTP GS $\geq 1$ symmetry & 55 & 79 & 3 & 0.56 & 73 & 64 & 0.672 \\
\hline MTP GS $\geq 2$ involvement & 59 & 72 & 2 & 0.57 & 68 & 64 & 0.655 \\
\hline MTP GS $\geq 2$ symmetry & 41 & 83 & 2 & 0.71 & 71 & 56 & 0.621 \\
\hline MTP PD $\geq 1$ involvement & 52 & 90 & 5 & 0.54 & 83 & 65 & 0.707 \\
\hline MTP PD $\geq 1$ symmetry & 35 & 100 & & 0.66 & 100 & 60 & 0.672 \\
\hline MTP PD $\geq 2$ involvement & 38 & 100 & & 0.62 & 100 & 62 & 0.690 \\
\hline MTP PD $\geq 2$ symmetry & 24 & 100 & & 0.76 & 100 & 57 & 0.621 \\
\hline \multicolumn{8}{|l|}{ Other variables } \\
\hline Leiden score $\geq 8$ & 62 & 93 & 9 & 0.41 & 90 & 71 & 0.776 \\
\hline 1987 ACR criteria (4/7 clinical) & 79 & 90 & 8 & 0.23 & 89 & 81 & 0.845 \\
\hline ACR criteria 4/7 GS & 93 & 65.5 & 3 & 0.10 & 73 & 91 & 0.793 \\
\hline ACR criteria 4/7 PD & 86 & 76 & 4 & 0.18 & 78 & 85 & 0.810 \\
\hline X-ray hand/foot erosion & 3.5 & 100 & & 0.97 & 100 & 45 & 0.517 \\
\hline US hand/foot erosion & 38 & 93 & 5.5 & 0.67 & 85 & 60 & 0.655 \\
\hline ACPA positive & 48 & 100 & & 0.52 & 100 & 66 & 0.741 \\
\hline $\begin{array}{l}\text { ACPA positive or MTP PD } \\
\geq 2 \text { Involvement or MCP GS count } \geq 8\end{array}$ & 76 & 100 & & 0.24 & 100 & 81 & 0.879 \\
\hline PD10 index $\geq 10$ & 79 & 93 & 11.5 & 0.22 & 92 & 82 & 0.862 \\
\hline
\end{tabular}

ACPA, anti-cyclic citrullinated peptide antibody; ACR, American College of Rheumatology; AUC, area under the receiver operating characteristic curve; GS, ultrasound greyscale; LR, likelihood ratio; metacarpophalangeal joint (MCP) GS $\geq 1$ involvement, MCP joint involvement with an ultrasound greyscale grade of at least 1; MCP joint GS count $\geq 8$, at least eight MCP joints with GS involvement of grade $\geq 1$; NPV, negative predictive value; PD, ultrasound power Doppler; PD10 index, summed power Doppler grades of MCP 2-3 joints, wrists and metatarsophalangeal (MTP) 2-3 joints; PIP, proximal interphalangeal joint; PPV, positive predictive value; RA, rheumatoid arthritis; US, ultrasound.

Doppler indices to 12 joints in the manner of Naredo et a ${ }^{24}$ had the effect of increasing the AUC, suggesting that joints effective for monitoring disease activity also have good specificity as predictors for RA. Removing the knee joint from this index further improved the AUC, and has the advantage of reducing scanning time. This finding requires validation in larger studies.

The increased sensitivity of ultrasound for erosions was greater than that in the ESPOIR cohort ${ }^{31}$ and of a similar order 
Table 4 Impact of ultrasound on prediction of RA: multivariate analyses

\begin{tabular}{|c|c|c|}
\hline Variables & p Value & AUC \\
\hline Leiden score & $<0.001$ & 0.905 \\
\hline \multicolumn{3}{|l|}{ Global US variables } \\
\hline Leiden & $0.005\}$ & \multirow{2}{*}{0.939} \\
\hline GS index & $0.028 \mathrm{~J}$ & \\
\hline Leiden & $0.003\}$ & \multirow{2}{*}{0.924} \\
\hline GS count & 0.078 S & \\
\hline Leiden & $0.007\}$ & \multirow{2}{*}{0.951} \\
\hline PD index & $0.016 \mathrm{~J}$ & \\
\hline Leiden & $0.006\}$ & \multirow{2}{*}{0.942} \\
\hline PD count & $0.025 \int$ & \\
\hline Leiden & $0.005\}$ & \multirow{2}{*}{0.952} \\
\hline PD12 index (after Naredo et al) ${ }^{24}$ & $0.010\}$ & \\
\hline Leiden & $0.007\}$ & \multirow{2}{*}{0.962} \\
\hline PD10 index & $0.006 J$ & \\
\hline \multicolumn{3}{|l|}{ US joint counts by region } \\
\hline Leiden & $0.003\}$ & \multirow{2}{*}{0.939} \\
\hline PD hand count & $0.034 \mathrm{~J}$ & \\
\hline Leiden & $0.002\}$ & \multirow{2}{*}{0.936} \\
\hline PD MCP count & 0.023 J & \\
\hline Leiden & $<0.001\}$ & \multirow{2}{*}{0.926} \\
\hline PD PIP count & 0.148 & \\
\hline Leiden & $<0.001\}$ & \multirow{2}{*}{0.929} \\
\hline PD MTP count & 0.080 S & \\
\hline Leiden & $0.005\}$ & \multirow{2}{*}{0.939} \\
\hline GS hand count & $0.030 \mathrm{~J}$ & \\
\hline Leiden & $0.004\}$ & \multirow{2}{*}{0.943} \\
\hline GS MCP count & $0.016 \mathrm{~J}$ & \\
\hline Leiden & $<0.001\}$ & \multirow{2}{*}{0.920} \\
\hline GS PIP count & 0.175 & \\
\hline Leiden & $<0.001\}$ & \multirow{2}{*}{0.905} \\
\hline GS MTP count & $0.547 \int$ & \\
\hline \multicolumn{3}{|l|}{ MCP joints } \\
\hline Leiden & $0.007\}$ & \multirow{2}{*}{0.930} \\
\hline MCP GS $\geq 1$ symmetry & $0.035 \int$ & \\
\hline Leiden & $0.002\}$ & \multirow{2}{*}{0.923} \\
\hline MCP GS $\geq 2$ symmetry & 0.049 & \\
\hline Leiden & $0.001\}$ & \multirow{2}{*}{0.932} \\
\hline MCP PD $\geq 2$ involvement & 0.034 & \\
\hline Leiden & $0.002\}$ & \multirow{2}{*}{0.937} \\
\hline MCP PD $\geq 2$ symmetry & 0.015 & \\
\hline \multicolumn{3}{|l|}{ Wrist joints } \\
\hline Leiden & $0.001\}$ & \multirow{2}{*}{0.936} \\
\hline Wrist GS $\geq 2$ involvement & 0.013 & \\
\hline Leiden & $<0.001\}$ & \\
\hline Wrist GS $\geq 2$ symmetry & $0.015 \zeta$ & 0.939 \\
\hline MTP joints & & \\
\hline Leiden & $0.001\}$ & \\
\hline MTP PD $\geq 1$ involvement & $0.026\}$ & 0.933 \\
\hline
\end{tabular}

AUC, area under the receiver operating characteristic curve; GS, ultrasound greyscale; GS count, sum of greyscale data when converted to a binary variable; GS index, sum of greyscale grades for all scanned joints; metacarpophalangeal (MCP) joint GS $\geq 1$ involvement, MCP involvement with an ultrasound GS grade of at least 1; $P D$, ultrasound power Doppler; PD10 index, summed power Doppler grades of MCP joint 2-3 joints, wrists and metatarsophalangeal (MTP) 2-3 joints; RA, rheumatoid arthritis; US, ultrasound.

of magnitude to that seen by Wakefield et al. ${ }^{14}$ Although not the strongest predictive variable in our analysis, ultrasound erosions had a high specificity for RA of $93 \%$, greater than that of radiographic erosions in the Leiden undifferentiated cohort $(77 \%) .32$ Sonographers should therefore remain assured that scanning for ultrasound erosions is of significant value in confirming clinically suspected disease. Although a recent study presented ultrasound examination of the fifth metatarsophalangeal joint as a useful test to confirm diagnosis in RA with a mean disease duration of 15 months, ${ }^{33}$ examining the fifth metatarsophalangeal joint

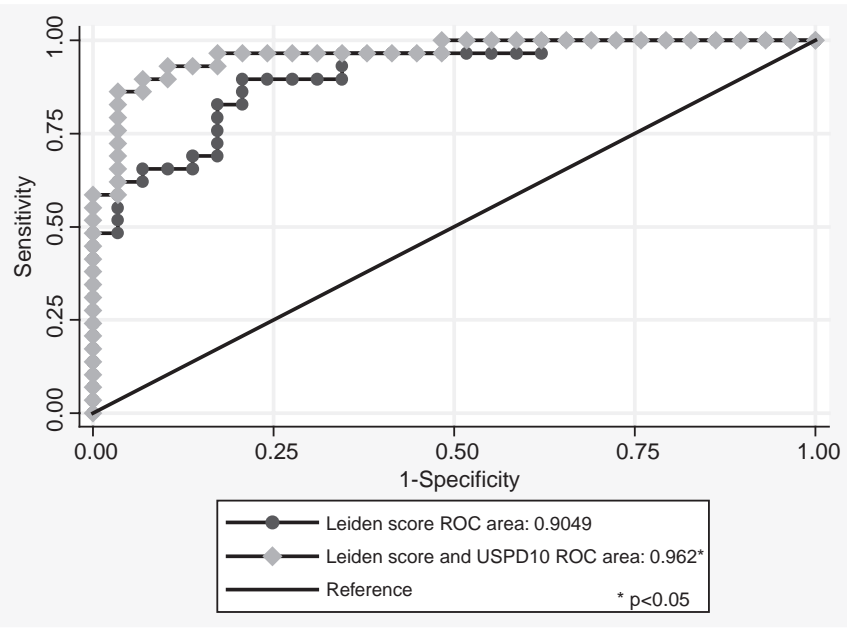

Figure 2 Area under the receiver operating characteristic (ROC) curve for rheumatoid arthritis (RA) as an outcome using a 10-joint power Doppler index (summed power Doppler grades of metacarpophalangeal joints 2-3, wrists and metatarsophalangeal 2-3 joints) combined with the Leiden score (grey), versus the Leiden score alone (black). The 10-joint power Doppler index was entered as an explanatory variable in logistic regression analysis with an outcome of rheumatoid arthritis RA as the dependent variable and the Leiden score as the independent variable. The area under the ROC curve was compared using a nonparametric algorithm developed by DeLong et al. ${ }^{25}$

for erosions in our very early cohort detected only one out of 29 RA patients This test is therefore not of use in the very early window of disease.

This study has some shortcomings that we have sought to minimise. The low proportion of female RA patients compared with a normal population may be a chance finding within a small group. Any subtle gender-related differences in RA severity ${ }^{34}$ are unlikely to impact on the results of this study. In addition, two patients with persistent unclassified disease that could potentially have developed into RA were treated with disease-modifying antirheumatic drugs. Our findings should be viewed with this in mind, as to omit treatment would have been unethical. We have harmonised any grading used with published schemes so as to maximise the applicability of our findings, and have taken precautions to eliminate sources of bias, for instance by using temperature controlled facilities to minimise power Doppler variability. The main limitation relates to the small size of this initial cohort. The sample size required to produce diagnostic algorithms using ultrasound measures of synovitis with unbiased statistical methods would be considerable, and data from the present study should inform the design of future studies. We have demonstrated that scanning not only the metacarpophalangeal joints, but also the wrist and metatarsophalangeal joints with greyscale and power Doppler, is likely to provide the optimum ultrasound data to improve on clinical predictive models for RA, and have demonstrated the unique predictive specificity of metatarsophalangeal joint sonography and power Doppler measurement for RA. These are vital first steps in the development of validated predictive algorithms that include ultrasound variables.

Funding The ultrasound equipment used in this study was funded by Arthritis Research UK, and the Rheumatology Research Group is a member of the EU AutoCure Consortium.

Competing interests $\mathrm{CDB}$ and $\mathrm{KR}$ have received grants and honoraria from Wyeth, Cellzome, UCB and Pfizer. AF has received grant support from Cellzome and Pfizer. SB has received honoraria or grant support from Roche, Genentech, UCB, 
GlaxoSmithKline and Astra-Zeneca. PdP, GA, PN, AJ and PJ declare no conflicts of interest.

Patient consent Obtained.

Ethics approval This study was conducted with the approval of the Solihull Local Research Ethics Committee.

Provenance and peer review Not commissioned; externally peer reviewed.

\section{REFERENCES}

1. Finckh $\mathbf{A}$, Liang $\mathrm{MH}$, van Herckenrode $\mathrm{CM}$, et al. Long-term impact of early treatment on radiographic progression in rheumatoid arthritis: a meta-analysis. Arthritis Rheum 2006;55:864-72.

2. Raza K, Buckley CE, Salmon M, et al. Treating very early rheumatoid arthritis. Best Pract Res Clin Rheumatol 2006;20:849-63.

3. Raza K, Falciani F, Curnow SJ, et al. Early rheumatoid arthritis is characterized by a distinct and transient synovial fluid cytokine profile of $\mathrm{T}$ cell and stromal cell origin. Arthritis Res Ther 2005; 7:R784-95.

4. Nell VP, Machold KP, Eberl G, et al. Benefit of very early referral and very early therapy with disease-modifying anti-rheumatic drugs in patients with early rheumatoid arthritis. Rheumatology (Oxford) 2004;43:906-14.

5. Möttönen T, Hannonen P, Korpela M, et al. Delay to institution of therapy and induction of remission using single-drug or combination-disease-modifying antirheumatic drug therapy in early rheumatoid arthritis. Arthritis Rheum 2002;46:894-8.

6. Raza K, Filer A. Predicting the development of RA in patients with early undifferentiated arthritis. Best Pract Res Clin Rheumatol 2009;23:25-36.

7. Arnett FC, Edworthy SM, Bloch DA, et al. The American Rheumatism Association 1987 revised criteria for the classification of rheumatoid arthritis. Arthritis Rheum 1988; 31:315-24

8. Aletaha D, Neogi T, Silman AJ, et al. 2010 Rheumatoid arthritis classification criteria: an American College of Rheumatology/European League Against Rheumatism collaborative initiative. Ann Rheum Dis 2010;69:1580-8.

9. van der Helm-van Mil AH, le Cessie S, van Dongen $\mathrm{H}$, et al. A prediction rule for disease outcome in patients with recent-onset undifferentiated arthritis: how to guide individual treatment decisions. Arthritis Rheum 2007;56:433-40.

10. van der Helm-van Mil AH, Detert J, le Cessie $\mathrm{S}$, et al. Validation of a prediction rule for disease outcome in patients with recent-onset undifferentiated arthritis: moving toward individualized treatment decision-making. Arthritis Rheum 2008; 58:2241-7.

11. Mjaavatten MD, Uhlig T, Haugen AJ, et al. Positive anti-citrullinated protein antibody status and small joint arthritis are consistent predictors of chronic disease in patients with very early arthritis: results from the NOR-VEAC cohort. Arthritis Res Ther 2009;11:R146

12. Szkudlarek M, Klarlund M, Narvestad E, et al. Ultrasonography of the metacarpophalangeal and proximal interphalangeal joints in rheumatoid arthritis: a comparison with magnetic resonance imaging, conventional radiography and clinical examination. Arthritis Res Ther 2006;8:R52

13. Wakefield RJ, Green MJ, Marzo-Ortega H, et al. Should oligoarthritis be reclassified? Ultrasound reveals a high prevalence of subclinical disease. Ann Rheum Dis 2004;63:382-5.

14. Wakefield RJ, Gibbon WW, Conaghan PG, et al. The value of sonography in the detection of bone erosions in patients with rheumatoid arthritis: a comparison with conventional radiography. Arthritis Rheum 2000;43:2762-70.

15. Freeston JE, Wakefield RJ, Conaghan PG, et al. A diagnostic algorithm for persistence of very early inflammatory arthritis: the utility of power Doppler ultrasound when added to conventional assessment tools. Ann Rheum Dis 2010;69:417-19.

16. Dougados M, Jousse-Joulin S, Mistretta F, et al. Evaluation of several ultrasonography scoring systems for synovitis and comparison to clinical examination: results from a prospective multicentre study of rheumatoid arthritis. Ann Rheum Dis 2010;69:828-33.

17. Raza K, Breese M, Nightingale P, et al. Predictive value of antibodies to cyclic citrullinated peptide in patients with very early inflammatory arthritis. J Rheumatol 2005; $32: 231-8$

18. Kingsley G, Sieper J. Third International Workshop on Reactive Arthritis, 23-26 September 1995, Berlin, Germany. Report and abstracts. Ann Rheum Dis 1996:55:564-84.

19. Gladman DD, Shuckett R, Russell ML, et al. Psoriatic arthritis (PSA) - an analysis of 220 patients. O J Med 1987:62:127-41.

20. Backhaus M, Burmester GR, Gerber T, et al. Guidelines for musculoskeletal ultrasound in rheumatology. Ann Rheum Dis 2001;60:641-9.

21. Wakefield RJ, Balint PV, Szkudlarek M, et al. Musculoskeletal ultrasound including definitions for ultrasonographic pathology. J Rheumatol 2005;32:2485-7.

22. Schmidt WA, Schmidt H, Schicke B, et al. Standard reference values for musculoskeletal ultrasonography. Ann Rheum Dis 2004;63:988-94.

23. Szkudlarek M, Court-Payen M, Jacobsen S, et al. Interobserver agreement in ultrasonography of the finger and toe joints in rheumatoid arthritis. Arthritis Rheum 2003:48:955-62.

24. Naredo E, Rodríguez M, Campos C, et al. Validity, reproducibility, and responsiveness of a twelve-joint simplified power doppler ultrasonographic assessment of joint inflammation in rheumatoid arthritis. Arthritis Rheum 2008;59:515-22.

25. DeLong ER, DeLong DM, Clarke-Pearson DL. Comparing the areas under two or more correlated receiver operating characteristic curves: a nonparametric approach. Biometrics 1988;44:837-45.

26. Harrison B, Symmons D. Early inflammatory polyarthritis: results from the Norfolk Arthritis Register with a review of the literature. II. Outcome at three years. Rheumatology (Oxford) 2000;39:939-49.

27. Thould AK, Simon G. Assessment of radiological changes in the hands and feet in rheumatoid arthritis. Their correlation with prognosis. Ann Rheum Dis 1966;25:220-8

28. Emery P, Breedveld FC, Dougados M, et al. Early referral recommendation for newly diagnosed rheumatoid arthritis: evidence based development of a clinical guide. Ann Rheum Dis 2002;61:290-7.

29. Taylor PC, Steuer A, Gruber J, et al. Comparison of ultrasonographic assessment of synovitis and joint vascularity with radiographic evaluation in a randomized, placebocontrolled study of infliximab therapy in early rheumatoid arthritis. Arthritis Rheum 2004;50:1107-16

30. Naredo E, Collado P, Cruz A, et al. Longitudinal power Doppler ultrasonographic assessment of joint inflammatory activity in early rheumatoid arthritis: predictive value in disease activity and radiologic progression. Arthritis Rheum 2007:57:116-24

31. Funck-Brentano T, Etchepare F, Joulin SJ, et al. Benefits of ultrasonography in the management of early arthritis: a cross-sectional study of baseline data from the ESPOIR cohort. Rheumatology (Oxford) 2009;48:1515-19

32. Thabet MM, Huizinga TW, van der Heijde DM, et al. The prognostic value of baseline erosions in undifferentiated arthritis. Arthritis Res Ther 2009;11:R155.

33. Sheane BJ, Beddy $\mathrm{P}, \mathrm{O}^{\prime}$ Connor M, et al. Targeted ultrasound of the fifth metatarsophalangeal joint in an early inflammatory arthritis cohort. Arthritis Rheum 2009;61:1004-8.

34. Sokka T, Toloza S, Cutolo M, et al. Women, men, and rheumatoid arthritis: analyses of disease activity, disease characteristics, and treatments in the QUEST-RA study. Arthritis Res Ther 2009:11:R7. 